\title{
Experimental results and analysis in terms of classical thermody- namics of variations in temperature and melting enthalpies in eutectic alloys upon changes in mutual phase orientation
}

\author{
A.M. Savchenko ${ }^{\dagger}$, Y.V. Konovalov, A.V. Laushkin \\ †sav-alex111@mail.ru \\ Stock Company «A.A. Bochvar High-Technology Research Institute of Inorganic Materials» (SC VNIINM), 123060, \\ Moscow, Russia
}

Experiments were carried out to validate the hypothesis of a possible rise in temperature of eutectic melting when mutual phase orientation becomes disarranged. Mutual disorientation of phases has to convert an eutectic alloy from the thermodynamic system state into a mechanical blend of phases. To disturb mutual phase orientation seven eutectic aluminium base alloys were rolled to different deformation degrees. Using differential thermal analysis method (DTA) temperature and enthalpy of melting were determined on the same samples in equilibrium and disorientation phase state. Increase of eutectic melting temperature up to $22^{\circ} \mathrm{C}$ and $7-14 \%$ reduce in enthalpy of melting were obtained. It was for the first time that the alloy was overheated to such a high temperature without a phase transformation at a relatively low heating rate $(80 \mathrm{deg} / \mathrm{min})$. This effect might be explained by the fact that free energy values of phases comprising eutectic are lower than in initial state when they are separated and does not depend on interphase surface energy. Thermodynamic equilibrium state of eutectics with lower free energy retains only at specific inter-phase orientation, which allow to provide valent electron correlations and metallic bond between eutectic phases. This in its turn lowers down the system energy and in terms of thermodynamics distinguishes an eutectic alloy from a mechanical blend of phases.

Keywords: melting, solidification, phases, thermodynamics, melting temperature.

\section{Анализ с позиции классической термодинамики эксперимен- тальных данных по изменению температуры и энтальпии плавления эвтектических сплавов при изменении взаимной ориентировки фаз}

\author{
Савченко А.М. ${ }^{\dagger}$, Коновалов Ю.В., Лаушкин А.В. \\ †sav-alex111@mail.ru \\ $\mathrm{AO}$ «Высокотехнологический научно-исследовательский институт неорганических материалов имени академика \\ А.А. Бочвара» (АО «ВНИИНМ»), 123060, г. Москва
}

Проведены эксперименты по проверке гипотезы о возможном увеличении температуры плавления эвтектики при на-
рушении взаимной ориентировки фаз. Нарушение взаимной ориентировки фаз должно переводить эвтектический
сплав из состояния термодинамической системы в механическую смесь фаз. Для нарушения взаимной ориентиров-
ки фаз семь эвтектических сплавов на основе алюминия были прокатаны с разной степенью деформации. Методом
дифференциального термического анализа (ДТА) на одних и тех же образцах определялась температура и энталь-
пия плавления эвтектических сплавов в равновесном состоянии и после нарушения взаимной ориентировки фаз.
Было получено увеличение температуры плавления до $22^{\circ} \mathrm{C}$, а также уменьшение энтальпии плавления на $7-14 \%$.
Впервые удалось перегреть сплав выше температуры плавления на столь большую температуру без прохождения
фазового превращения при относительно низкой скорости нагрева (80 град/мин). Данный эффект можно объяс-
нить тем, что величина свободной энергии фаз, составляющих эвтектику, меньше, чем у исходных фаз и не зависит
от межфазной поверхностной энергии. Термодинамическое равновесное состояние эвтектических сплавов сохраня- 
ется только при определенной взаимной ориентировке фаз в эвтектике, что позволяет осуществлять электронное взаимодействие и металлическую связь между фазами, ведущую к понижению энергии системы, а также понижению температуры плавления эвтектик, что термодинамически отличает эвтектический сплав от механической смеси фаз.

Ключевые слова: плавление, затвердевание, фазы, термодинамика, температура плавления.

\section{1. Введение}

Известно, что сплавы, особенно эвтектические, легко можно переохладить ниже температуры плавления без прохождения фазового превращения на десятки и сотни градусов, но пока не удавалось перегреть их хотя бы на градус выше температуры плавления даже при больших скоростях нагрева [1].

В настоящее время более низкие температуры плавления эвтектических сплавов (по сравнению с температурами плавления компонентов), согласно термодинамике сплавов, объясняются увеличением энтропии смешения в жидком состоянии, тем самым уменьшается свободная энергия расплава и жидкое состояние стабилизируется. Таким образом, в процессе кристаллизации даже при низких температурах сохраняется более высокоэнергетическое жидкое состояние исходных компонентов эвтектики, имеющих более высокую температуру плавления [2-3].

Однако не совсем ясно, каким образом происходит обратный процесс - плавление эвтектического сплава при более низкой температуре. Известно, что при эвтектической кристаллизации из расплава с перемешанными атомами А и В и преимущественно межатомными связями А-В образуются кристаллы АА и ВВ со связями A-А и В-B соответственно. Каким образом, при обратном нагреве из твердого состояния эти фазы плавятся при более низкой эвтектической температуре, если нет смешения атомов, то есть, нет связей А-В и отсутствует энтропия смешения? Каким образом смесь отдельных фаз уже «знает», что ей пора плавиться, и плавление происходит одновременно при одной температуре по всему объему? На этот вопрос термодинамика сплавов внятного объяснения не дает.

Сейчас считается, что эвтектический сплав представляет собой механическую смесь фаз АА и $\mathrm{BB}$ и плавление начинается по границам фаз, где атомы А и В непосредственно соприкасаются. Затем эта межграничная область должна мгновенно распространиться по всему объему сплава. Однако экспериментально установлено, что эвтектики как мелкозернистые, так и крупнозернистые, отличающиеся площадью межфазной поверхности в сотни раз, плавятся без перегрева по всему объему при одной и той же более низкой эвтектической температуре, даже при скорости нагрева $10^{6}$ град/сек.

Подход к фазовой смеси как к механической смеси фаз противоречит не только экспериментальным данным, но и классической термодинамике, которая всегда рассматривает любую многокомпонентную систему, в том числе и сплавы, как единую термодинамическую систему. Это подчеркивал А.А. Бочвар в своих работах по изучению особенностей кристаллизации эвтектических сплавов [4].

В проведенных нами ранее исследованиях [5] была высказана гипотеза, что одновременное плавление при эвтектической температуре фаз, имеющих в изолированном состоянии более высокие температуры плавления объясняется тем, что в эвтектиках, несмотря на наличие в структуре отдельных фаз АА и ВB, соответственно, между ними сохраняется связь А-В, характерная только для жидкого состояния. Эта связь проявляется в дополнительном расщеплении электронных уровней, соответствующих состоянию А-В. Так как этот тип связи не является следствием межфазного взаимодействия по границам зерен и фаз, а является внутренней связью между фазами, то ни структура эвтектики, ни протяженность границ зерен, по которым, согласно существующим теориям должно начинаться плавление, не влияют на температуру плавления.

Согласно гипотезе, эта связь, условно названная термодинамической, существует только при определенной взаимной ориентировке фаз, и в случае ее нарушения эвтектика, несмотря на сохранение мелкозернистой структуры, и металлургического сцепления между фазами, будет вести себя как механическая смесь фаз, то есть плавиться при более высокой температуре.

В данной статье сделана попытка экспериментальной проверки данной гипотезы. Проведен анализ полученных экспериментальных данных с позиций классической термодинамики.

\section{2. Экспериментальная часть}

Для проведения исследований было изготовлено 7 сплавов на основе алюминия систем $\mathrm{Al}-\mathrm{Si}, \mathrm{Al}-\mathrm{Cu}$, Al-Cu-Si, Al-Si-Ni, Al-Si-Ni-Fe, Al-Mg-Si-Cu, Al-Si-Mg-Cu (см. табл. 1). Были выбраны эвтектические относительно легкоплавкие сплавы на основе алюминия из соображения технологичности получения сплава и проведения прокатки.

Для нарушения термодинамической связи исходные образцы сплавов были прокатаны в холодную с разной степенью деформации. При прокатке частично менялась ориентировка фаз, и термодинамическая связь (внутренняя металлическая А-В связь, выраженная дополнительным расщеплением уровней валентных электронов, соответствующих состоянию А-В) в некоторых местах нарушалась. При этом металлография не выявила дефектов, приводящих к нарушению металлургического сцепления между фазами (рис. 1).

Температуры и энтальпии плавления сплавов определяли методом дифференциального термического анализа (ДТА) на приборе ВДТА-8М в атмосфере гелия после вакуумирования. Скорость нагрева и охлаждения составляла 80 град/мин. Внутри прибора помещался термостат из чистого вольфрама, в отверстиях которого размещались тигли из танталовой фольги толщиной 0.2 мм с образцом и эталоном из вольфрама. Тигли при- 
Табл. 1. Результаты измерений температур и энтальпий плавления эвтектических сплавов на основе алюминия Table 1. Results of Measuring Melting Temperatures and Enthalpies of Al Base Eutectic Alloys

\begin{tabular}{|c|c|c|c|c|}
\hline $\begin{array}{c}\text { Состав сплавов, } \\
\% \text { масс. } \\
\text { Composition, } \\
\% \text { mass }\end{array}$ & $\begin{array}{c}\text { Фазовый состав } \\
\text { Phase composition }\end{array}$ & $\begin{array}{c}\text { Степень дефор- } \\
\text { мации, \% } \\
\text { Deformation } \\
\text { degree, \% }\end{array}$ & \begin{tabular}{|c|} 
Увеличение \\
температуры \\
плавления после \\
деформации, ${ }^{\circ} \mathrm{C}$ \\
Melting \\
temperature rise \\
after deformation, ${ }^{\circ} \mathrm{C}$
\end{tabular} & $\begin{array}{c}\text { Уменьшение } \\
\text { энтальпии } \\
\text { плавления после } \\
\text { деформации, \% } \\
\text { Decrease of enthalpy } \\
\text { of melting after } \\
\text { deformation, \% }\end{array}$ \\
\hline $\mathrm{Al}$ & $\mathrm{Al}^{*}$ & 90 & 0 & 1.5 \\
\hline $\mathrm{Al}-11.5 \mathrm{Si}$ & $\mathrm{Al}^{*}+\mathrm{Si}^{* *}$ & 90 & 4 & 11.2 \\
\hline $\mathrm{Al}-33 \mathrm{Cu}$ & $\mathrm{Al}^{\star}+\mathrm{CuAl}_{2}$ & 4 & 9 & 8.1 \\
\hline $\mathrm{Al}-26 \mathrm{Cu}-5.2 \mathrm{Si}$ & $\mathrm{Al}^{\star}+\mathrm{CuAl}_{2}+\mathrm{Si}$ & 90 & 0 & 8.3 \\
\hline Al-11.8Si-5Ni & $\mathrm{Al}^{\star}+\mathrm{Si}^{* *}+\mathrm{NiAl}_{3}$ & 90 & 3 & 10.1 \\
\hline Al-11Si-4Ni-1Fe & $\begin{array}{c}\mathrm{Al}^{*}+\mathrm{Si}^{* *}+\mathrm{NiAl}_{3}+ \\
(\mathrm{Fe}, \mathrm{Ni}) \mathrm{Al}_{9}\end{array}$ & 90 & 0 & 8.4 \\
\hline Al-9.2Mg-4.75Si-3Cu & $\begin{array}{c}\mathrm{Al}^{\star}+\mathrm{Mg}_{2} \mathrm{Si}+ \\
\mathrm{Al}_{2}(\mathrm{Cu}, \mathrm{Mg})\end{array}$ & 82 & 12 & 14.3 \\
\hline Al-14.3Si-5Mg-4Cu & $\begin{array}{c}\mathrm{Al}^{\star}+\mathrm{Si}^{* *}+ \\
\mathrm{Al}_{4} \mathrm{CuMg}_{5} \mathrm{Si}_{4}+\mathrm{CuAl}_{2}\end{array}$ & 64 & 22 & 7.3 \\
\hline
\end{tabular}

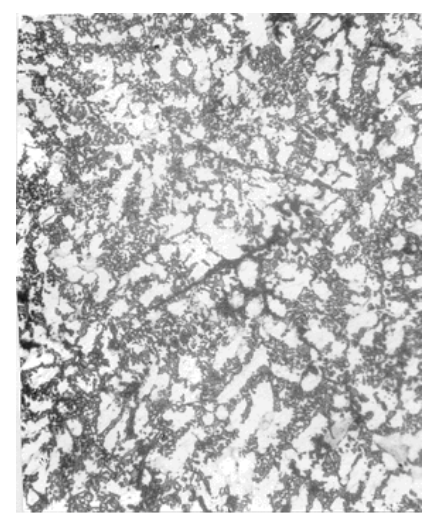

a

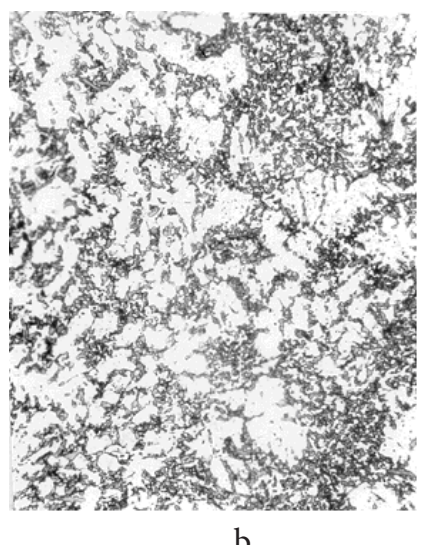

b
Рис. 1. Микроструктура $\mathrm{Al}-\mathrm{Si}-\mathrm{Mg}-\mathrm{Cu}$ сплава до (а) и после (b) прокатки, $\times 420$.

Fig. 1. Microstructure of Al-Si-Mg-Cu alloy before (a) and after (b) rolling, $\times 420$.

соединялись непосредственно к W-Re термопаре [6]. За счет использования тиглей из танталовой фольги точность измерений была высокой и составляла $\pm 1^{\circ} \mathrm{C}$.

Результаты экспериментов приведены на рис. 2.

Прокатанный образец алюминиевого сплава с частично нарушенной термодинамической связью в процессе ДТА нагревался до плавления (сплошная линия на рис. $2 \mathrm{a}, 2 \mathrm{~b}, 2 \mathrm{~d})$. После плавления нарушенная взаимная ориентировка фаз восстанавливалась и образец тем самым возвращался в исходное структурное состояние, каким он был до прокатки. Затем, не вынимая образец из прибора, его снова (повторно) подвергали
ДТА уже со структурой исходного образца до прокатки с восстановленной взаимной ориентировкой фаз и, следовательно, в исходном равновесном термодинамическом состоянии (пунктирная линия на рис. $2 \mathrm{a}, 2 \mathrm{~b}, 2 \mathrm{~d}$ ). Тем самым достигалась чистота и точность эксперимента, так как ДТА проводился на одном и том же образце, но в разном термодинамическом состоянии. Такая методика испытаний позволила повысить точность измерений разности температур до $\pm 1^{\circ} \mathrm{C}$ и разницы величин энтальпий плавления до $\pm 1 \%$. Подтверждением этого могут служить полностью совпадающие кривые охлаждения при ДТА прокатанных и исходных образцов, так как после расплавления сплавы переходили в равновесное состояние (рис. 2c). Для дополнительной оценки погрешности метода измерения проводился третий нагрев того же самого образца. Кривые третьего нагрева полностью совпадали с кривыми второго нагрева.

Следует отметить, что взаимодействия образцов с тиглем не было, и форма прокатанных пластинок после ДТА не менялась, так как тонкие окисные пленки на поверхности пластин алюминиевых сплавов не позволяли сплаву растекаться по тиглю при небольших перегревах выше температуры плавления.

Так как любая деформация повышает энергию системы, что при плавлении может привести к уменьшению энтальпии плавления, то для чистоты экспериментов учитывался вклад, который может вносить энергия деформации в виде дефектов кристаллической решетки. Для этого по той же схеме с деформацией 90\% прокатывался образец из чистого алюминия, который структурно составляет пластичную основу исследуемых сплавов. 


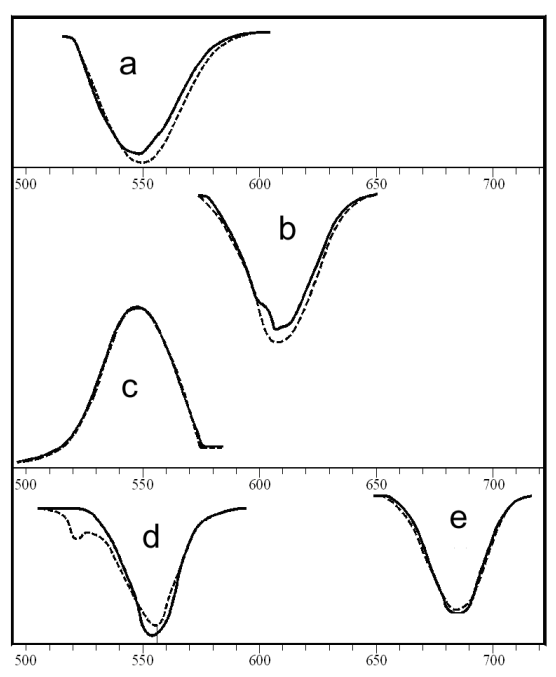

Рис. 2. Дифференциальный термический анализ эвтектических сплавов и алюминия: сплошная линия - образцы после прокатки (первый нагрев), пунктирная линия переплавленные образцы (в исходном состоянии - второй нагрев): Al-Cu-Si нагрев (a); Al-Si нагрев (b); Al-Si охлаждение (c); $\mathrm{Al}-\mathrm{Si}-\mathrm{Mg}-\mathrm{Cu}$ нагрев (d); $\mathrm{Al}$ нагрев (e).

Fig. 2. Differential thermal analysis of the eutectic alloys: solid line - rolled samples (first heating), dotted line - remelted samples (original state - second heating): Al-Cu-Si heating (a); Al-Si heating (b); Al-Si cooling (c); Al-Si-Mg-Cu heating (d); Al heating (e).

\section{3. Результаты}

На контрольном образце из чистого алюминия после прокатки температура плавления не изменилась, а энтальпия плавления уменьшилась только на $1.5 \%$, что составляет вклад энергии деформации (табл. 1, рис. 2е). По-видимому, даже относительно низкая скорость нагрева (80 град/мин) является достаточной высокой, и рекомбинация дефектов кристаллической решетки пройти не успевает. Основное изменение происходит в начале плавления, когда с появлением первой жидкой фазы почти сразу же проходит рекомбинация дефектов кристаллической решетки.

Что касается образцов алюминиевых эвтектических сплавов, то для них характер плавления качественно отличался.

После деформации произошло изменение (уменьшение) энтальпии плавления, в среднем на $7-14 \%$ в зависимости от сплава, количественно на 1 кДж/моль. Причем этот эффект происходил в конце плавления сплавов, когда происходит процесс смешения в жидком состоянии. Сначала, при температуре плавления эвтектики, плавилась неповрежденная деформацией часть эвтектики, а разориентированные в процессе деформации фазы оставались еще в твердом состоянии, так как их температура плавления после деформации стала выше. При дальнейшем повышении температуры их растворение в первичном расплаве проходило с выделением тепловой энергии смешения, так как они представляли собой простую механическую смесь, и не являлись термодинамически связанной частью эвтектики. Изломы кривых (см. рис. 2b, 2d) показывают начало этого процесса. Выделение энергии при смешении уменьшило ве- личину энтальпии плавления. Причем это произошло, как было отмечено выше, в конце плавления (рис. 2a, 2b).

У большинства сплавов увеличилась также и температура плавления эвтектики, что является принципиально новым результатом (табл. 1, рис. 2). Если сплавы были строго эвтектического состава, то увеличение температуры плавления составляло $3-9^{\circ} \mathrm{C}$ (сплавы $\mathrm{Al}-\mathrm{Si}$, $\mathrm{Al}-\mathrm{Cu}, \mathrm{Al}-\mathrm{Si}-\mathrm{Ni}$ ), если они состояли из нескольких эвтектик (сплавы $\mathrm{Al}-\mathrm{Mg}-\mathrm{Si}-\mathrm{Cu}$ ), то температура плавления увеличивалась на $12-22^{\circ} \mathrm{C}$.

\section{4. Обсуждение результатов}

По существующим в настоящее время традиционным термодинамическим описаниям эвтектики как механической смеси фаз взаимная ориентировка фаз в эвтектике не влияет на процесс плавления - ни на энтальпию плавления, ни на температуру плавления сплавов [2 -3]. Однако это не подтверждалось в наших экспериментах. Полученное увеличение температуры плавления и изменение энтальпии плавления сплавов на величину, превышающую влияние энергии деформации, показало, что произошло изменение термодинамического состояния эвтектических сплавов.

Следует отметить, что описанное нами поведение эвтектик не связано с метастабильной микрогетерогенностью эвтектических расплавов [7-8], так как оно наблюдается в твердом состоянии и при плавлении оно исчезает.

Если предположить в соответствии с классической термодинамикой и подходом А.А. Бочвара [4], что эвтектика является единой термодинамической системой, то при ее образовании должна уменьшаться свободная энергия системы, что не принимается во внимание при описании эвтектик традиционной геометрической термодинамикой (рис. 3, сплошные черные линии) [9]. Рассмотрим фазовое равновесие для составов 1, 2 и 3 , содержащих эвтектику, ниже температуры плавления. Сплавы имеют в структуре одни и те же фазы $\alpha$ и $\beta$, но в разном объемном соотношении. При традиционном описании фазового равновесия методом геометрической термодинамики, когда за основу берется механическая смесь фаз, представленная точками 1, 2 и 3, невозможно получить диффузией сплав эвтектического состава 1 из доэвтектического сплава 2 и заэвтектического сплава 3 , так как при этом не происходит уменьшение свободной энергии (сплошная черная линия на рис. 3). Однако реально диффузия происходит, так как величина свободной энергии образующегося сплава при этом понижается и должна описываться различными пунктирными линиями для сплавов 1,2 и 3 (рис. 3). Тогда очевидно, что при получении из сплавов 2 и 3 сплава состава 1 величина свободной энергии, в соответствии с классической термодинамикой понижается (точка 1' лежит ниже точек 2' и $3^{\prime}$ ) и процесс диффузии идет.

Жидкость эвтектического состава плавится при более низкой температуре за счет увеличения энтропии смешения, и, следовательно, уменьшения свободной энергии по сравнению с исходными компонентами в соответствии со вторым законом термодинамики [2-3]. 
Мы предполагаем, что энтропия смешения имеет энергетический смысл и даже может производить работу создавать осмотическое давление.

Увеличение энтропии приводит к увеличению амплитуды колебаний атомов и снижению температуры Дебая [9]. При кристаллизации эвтектики, когда из гомогенной смеси фазы разделяются и энтропия смешения формально исчезает, то реально ее энергия трансформируется в увеличение амплитуды колебаний атомов, то есть в обычную вибрационную энтропию, которая, в свою очередь увеличивает среднюю теплоемкость сплава по сравнению с механической смесью фаз [9].

Таким образом, можно предположить, что в эвтектике кроме связей А-А и В-B, сохраняется внутренняя связь А-В, характерная только для жидкого состояния, приводящая к понижению термодинамической энергии системы.

Из-за наличия этой связи, условно названной термодинамической, эвтектические сплавы, независимо от структуры, плавятся всегда при одной и той же температуре.

Термодинамическая связь влияет на характер и энергию колебаний атомов, что приводит к уменьшению температуры плавления эвтектик.

Так как эта связь, подобно металлической связи в металлах, выражается в вырождении и расширении атомных энергетических уровней валентных электронов и распространяется на всю глубину фаз, то она может существовать только при определенной ориентировке фаз.

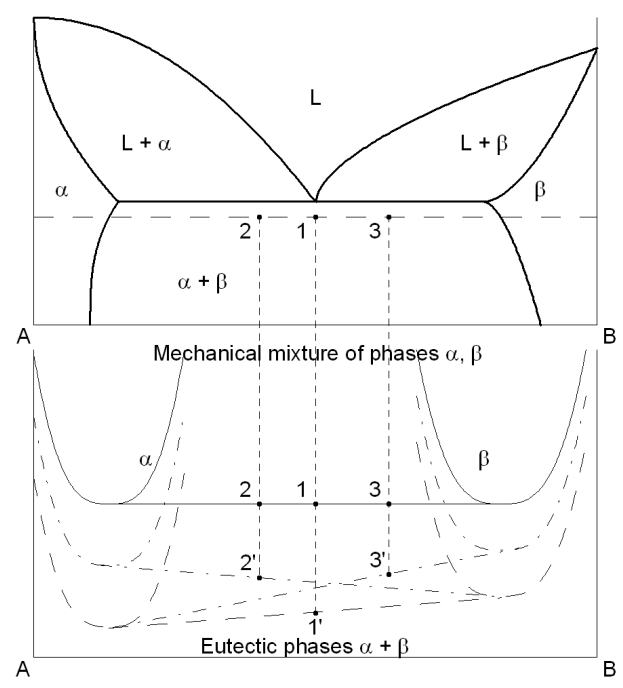

Рис. 3. Описание равновесия в эвтектической системе: традиционное - (сплошная черная линия, усредненное приведенное значение) - c использованием величин свободных энергий изолированных фаз (механическая смесь фазовое равновесие не зависит от состава); предлагаемая в соответствии с классической термодинамикой (пунктирные линии для сплавов 1', 2' и 3') - с использованием кривых свободных энергий фаз в термодинамическом контакте друг с другом - фазовое равновесие зависит от состава.

Fig. 3. Description of equilibrium in eutectic: traditional - (solid black line - reduced average value) - using free energies of isolated phases in an alloy (mechanical mixture - phase equilibrium does not depend on composition); Proposed in accordance with classical thermodynamics - (dotted lines for alloys 1', 2' and 3') - using free energies of phases contacting each other in an alloy - phase equilibrium depends on composition.

\section{5. Выводы}

Впервые удалось перегреть эвтектические сплавы выше температуры плавления без прохождения фазового превращения при относительно низкой скорости нагрева (80 град/мин). Это явление хорошо объясняется, если рассматривать эвтектику не как механическую смесь фаз, а как единую термодинамическую систему, в соответствии с классической термодинамикой и научным подходом А.А. Бочвара. В этом случае можно предположить образование дополнительной А-В связи в эвтектике в твердом состоянии между фазами, подобно металлической, когда валентные электроны одной фазы взаимодействуют с валентными электронами другой эвтектической фазы через взаимно ориентированные межфазные границы. В результате энергия системы понижается.

Обнаруженное новое явление требует дальнейшего изучения и экспериментального подтверждения на различных типах сплавов, в том числе и на простых эвтектиках без интерметаллических соединений.

\section{Литература/References}

1. A.I. Somov, M.A. Tikhonovsky. Eutectic Compositions. Moscow, Metallurgy (1975) 304 p. (in Russian) [А.И.Сомов, М.А Тихоновский. Эвтектические композиции. М.: Металлургия, 1975. 304 с.]

2. A.H. Cottrell Theoretical Structural Metallurgy. Arnold London. (1948) $256 \mathrm{p}$.

3. I. Prigogine, R. Defay. Chemical Thermodynamics. Wiley. (1954) 543 p.

4. A.A. Bochvar. Metal Science, Moscow, Metallurgy, (1956) 494 р. [Бочвар А.А. Металловедение, М., Металлургиздат, 1956. 494 с.]

5. A.M. Savchenko, Y.V. Konovalov, O.I Uferov. Journal of Physical and Chemical Materials Treatment. 3, 5-14 (2005), (in Russian) [А.М. Савченко, Ю.В. Коновалов, О.И Юферов. Физика и химия обработки материалов. 3. 5-14 (2005)]

6. A.M. Savchenko, N.I. Nogin, O.I. Uferov. Issues of Atomic Science and Engineering. Materials Science and Novel Materials. 2(73), 18-24 (2012), (in Russian) [A.M. Савченко, О.И. Юферов, Н.И. Ногин. Вопросы атомной науки и техники. Материаловедение и новые материалы. 2(73). 44-51 (2012)]

7. P.S. Popel, M. Calvo-Dahlborg, U. Dahlborg. Metastable microheterogeneity of melts in eutectic and monotectic systems and its influence on the properties of the solidified alloy. Journal of Non-Crystalline Solids. 353, 3243-3253 (2007)

8. D. Yagodin, P. Popel, V. Filippov. Metastable microheterogeneity of melts in the $\mathrm{Ga}-\mathrm{Bi}$ system with limited solubility of components in liquid state. Journal of Material Science. 45, 2035-2041 (2010)

9. A.M. Savchenko, A.V. Laushkin, P. Turchi, M. Fluss. In: Proceedings of Plutonium Futures 2012 - the Science Conference, University of Cambridge, UK, 15-10 July 2012 